\title{
La comprensión lectora de escolares de educación básica*
}

\author{
José Luis Gallego Ortega* \\ Susana Figueroa Sepúlveda"* \\ Antonio Rodríguez Fuentes
}

\section{Resumen}

El principal objetivo de esta investigación fue conocer el nivel de comprensión lectora de una muestra de estudiantes chilenos, escolarizados en un centro urbano subvencionado. Mediante un estudio transversal de corte cuantitativo, fue evaluado un total de 186 alumnos (95 niñas y 91 niños) de segundo año a octavo año de educación básica, para conocer su nivel de comprensión lectora (literal, inferencial y crítico) y su nivel de aprendizaje lector (insuficiente, elemental y adecuado). Los resultados revelaron que el nivel de comprensión lectora y, por tanto, su aprendizaje, se empobrece a medida que los estudiantes avanzan de curso escolar, comprobándose una progresión inadecuada en cuanto al desarrollo de la lectura, así como la inexistencia de diferencias significativas por razón de género.

Palabras clave: evaluación, lectura, lectura oral.

\section{Reading comprehension of basic education students}

\begin{abstract}
The acquisition of new knowledge depends on the reading. The main objective of this research was to know the level of reading comprehension of a sample of Chilean students educated in a financed urban school. Through a quantitative and transverse study a total of 186 students ( 95 girls and 91 boys) from the $2^{\text {nd }}$ to the $8^{\text {th }}$ course of basic education were evaluated in order to know their level of reading comprehension (literal, deductible and critical) and their level of reading learning (insufficient, elementary and appropriate). The results of this research show that the level of reading comprehension and, consequently, its learning, decreases as far as the students progress in the school year, bringing to light an inappropriate progression with regards to the reading development, as well as that there are no significant differences because of the sex.
\end{abstract}

Keywords: evaluation, oral reading, reading.

\footnotetext{
* Este artículo forma parte de una línea de investigación en torno a la didáctica, la lectura y la escritura que el autor principal ha desarrollado desde hace más de dos décadas

** Español. Doctor en Ciencias de la Educación. Académico de la Universidad de Granada, Granada, España.jlgalleg@ugr.es

*** Chilena. Doctora en Ciencias de la Educación de la Universidad de Granada. Académica de la Universidad Arturo Prat, Iquique, Chile.susfigue@unap.cl

**** Español. Doctor en Ciencias de la Educación. Académico de la Universidad de Granada, Granada, España. arfuente@ugr.es
} 


\section{Introducción}

La lectura desempeña un importante papel en el desarrollo de los procesos psicológicos superiores (Vygotsky, 1979), así como en el aprendizaje (Dreyer \& Nel, 2013). De hecho, uno de los principales objetivos de la educación básica es conseguir que los estudiantes adquieran habilidades y estrategias suficientes para ser competentes en tareas de lectura y escritura, siendo el aprendizaje lecto-escritor una aspiración constante de cualquier sistema educativo. Sin embargo, las investigaciones revelan las numerosas dificultades que exhibe el alumnado de educación básica en el aprendizaje de la lengua escrita, considerando el predominio que han tenido los métodos tradicionales en su enseñanza-aprendizaje, lo que ha contribuido a desfigurar la idea de lo que significa leer y escribir (Jurado, Sánchez, Cerchiaro y Paba, 2013).

Desde una perspectiva cognitiva, se asume que la lectura se ejecuta por un procesamiento en distintos niveles, que abarca desde procesos básicos de percepción de grafemas, conversión grafema-fonema, reconocimiento de palabras y asignación de funciones sintácticas a las palabras que componen la oración, hasta procesos de mayor nivel como la integración del significado de las oraciones que forman un discurso y la realización de inferencias (Van den Broeck, Geudens \& Van den Bos, 2010), las cuales son importantes para comprender el mundo (Dubois, 1984). Por su parte, la lectura demanda comprensión e interpretación personal de un texto, lo cual exige activar estrategias de alto nivel cognitivo que van más allá del simple descifrado mecánico de palabras o frases. Entonces, poseer competencia lectora significa "comprender, utilizar, reflexionar y comprometerse con textos escritos para alcanzar los propios objetivos, desarrollar el conocimiento y potencial personales y participar en la sociedad" (Organisation for Economic Co-operation and Development, OECD, 2015, p. 9), con lo que se intenta subrayar el sentido pragmático de la lectura y la capacidad del lector para aplicar el conocimiento adquirido mediante la lectura. Ciertamente, los términos comprensión lectora (reading comprehension) y competencia lectora (reading literacy) no son sinónimos totales, si bien en este texto se utilizan de forma unívoca ${ }^{1}$.

Para una revisión respecto de esta distinción se puede consultar Jiménez (2014). 
El lector experto maneja diferentes habilidades para reconocer y nombrar las palabras, así como para construir activamente los significados de un texto, involucrando simultáneamente los conocimientos lingüísticos, las ideas previas, el razonamiento o el conocimiento del mundo. Más que una interpretación de fonemas y el reconocimiento de palabras, entonces, la lectura demanda anticipación, inferencia, predicción y creación; es traspasar el significado de un texto para comprender su complejidad. Leer exige decodificar, pero también comprender, en la medida en que se trata de un proceso constructivo e integrador que se produce en la interacción entre el lector, el texto y el contexto (Alliende y Condemarín, 2002; Guzmán, Véliz y Reyes, 2017; Neira, Reyes y Riffo, 2015).

La importancia de este aprendizaje — clave para el éxito escolar-, ha llevado a profesionales e investigadores a mantener el interés en torno a las dificultades que influyen en el proceso lector, para tratar de implementar estrategias que permitan satisfacer los diferentes condicionantes que pueden afectar el rendimiento de los alumnos (Wise, D’Angelo \& Chen, 2016). Desde esta perspectiva, numerosos estudios realizados acerca del desempeño del alumnado de educación básica en tareas de lectura han puesto de manifiesto diferentes hallazgos en relación con los tres procesos básicos implicados: decodificación, comprensión y metacomprensión. Se sabe, por ejemplo, de la correlación que existe entre conciencia fonológica y competencia lectora (Couceiro \& Botelho, 2017; De la Calle, Villagrán y Guzmán, 2016), entre la decodificación y la comprensión lectora (Bigozzi, Tarchi, Vagnoli, Valente \& Pinto, 2017), o entre el léxico y la comprensión lectora (Perfetti \& Stafura, 2014; Riffo, Reyes, Novoa, Véliz y Castro, 2014), así como la relación significativa entre la comprensión lectora y la automatización de los procesos de bajo nivel (Outón y Suárez, 2011). Igualmente, se conoce la influencia de la escolarización temprana y el nivel sociocultural en la comprensión lectora (González y López, 2015), o la mejor comprensión por parte del alumnado de los textos narrativos que los expositivos (Best, Floyd \& McNamara, 2008). Tampoco se ignora que la comprensión verbal correlaciona con la comprensión lectora (Bohórquez, Cabal y Quijano, 2014), ni que las habilidades metalingüísticas (conciencia fonológica y sintáctica) evolucionan durante la educación básica (Mariángel y Jiménez, 2016), o la necesidad de ahondar en el conocimiento de la relación entre la comprensión lectora y la metacognición (Ramírez, 
Rossel y Nazar, 2016), considerando que la enseñanza de estrategias de comprensión y metacomprensión se perfila como un remedio eficaz para la comprensión de textos (Rodríguez, Calderón, Leal y Arias, 2016).

Sin embargo, son testimoniales los estudios cuyo foco de atención exclusivo es la evaluación de la comprensión lectora del alumnado para dar cuenta de su nivel de logro (Alonso, Carriedo, González, Gutiérrez y Mateos, 1992; Pérez-Zorrilla, 1998), aunque la preocupación por conocer la competencia de los estudiantes en lectura no es nueva y es tarea común de organismos internacionales, autoridades educativas, profesionales e investigadores evaluar el nivel de comprensión lectora del alumnado, a partir de diversos procedimientos (pruebas estandarizadas y no estandarizadas), teniendo presente la complejidad que entraña esta labor y la multiplicidad de instrumentos existentes.

En la comprensión lectora se distinguen tres niveles (Smith, 1989): literal, que se refiere a la habilidad del alumno para comprender explícitamente lo dicho en el texto; inferencial, que indica la habilidad del estudiante para elaborar conjeturas e hipótesis acerca del texto; crítico, que se vincula con su habilidad para evaluar la calidad de un texto y la emisión de juicios razonados sobre el mismo.

En Chile, al tenor de los resultados obtenidos en las pruebas Simce (Sistema de Medición de la Calidad de la Educación) que se aplican cada año a alguno de los niveles de enseñanza, en general se ha constatado un bajo nivel de comprensión lectora entre el alumnado de educación básica. El Ministerio de Educación chileno (Mineduc) entiende que “comprender un texto implica extraer información, inferir o interpretar aspectos que no están expresamente dichos, y evaluarlos críticamente. Esto supone que el lector asume un papel activo, relacionando sus conocimientos previos con los mensajes que descubre en la lectura" (Mineduc, 2012, p. 36). A este efecto, el Mineduc establece, a partir de los niveles de comprensión lectora alcanzados por el alumnado, otros tantos niveles de aprendizaje según estándares de aprendizaje, es decir, de acuerdo con "referentes que describen lo que los estudiantes deben saber y poder hacer para demostrar, en las evaluaciones Simce, determinados niveles de cumplimiento de los objetivos de aprendizaje estipulados en el currículo vigente" (Mineduc, 2014, p. 4), entre los que destaca: nivel adecuado, cuando el alumnado satisface las exigencias establecidas en el currículo; nivel elemental, cuando el alumnado logra parcialmente los requerimientos curriculares; nivel insuficiente, cuando el alumnado 
no demuestra consistentemente la adquisición de conocimientos y habilidades más elementales acordados en el currículo oficial.

En suma, indagar en los procesos que subyacen en la comprensión de textos sigue siendo una preocupación en el ámbito de la investigación educativa, a la cual corresponde desvelar cuáles son las principales dificultades lectoras del alumnado para buscar posibles soluciones. No en vano entre el $3 \%$ y el $10 \%$ de los niños en edad escolar muestran problemas en lectura (Goswami, 2011).

En este sentido, los objetivos de esta investigación fueron:

- Identificar los niveles de comprensión lectora del alumnado de $2^{\circ} \mathrm{a}$ $8^{\circ}$ año básico, obteniendo significatividad estadística.

- Analizar las posibles diferencias intracaso e intercaso, estimando las discrepancias entre el alumnado de los distintos cursos y del mismo curso.

- Proporcionar orientaciones didácticas para mejorar la comprensión lectora.

\section{Método}

La investigación consistió en un estudio descriptivo y transversal de corte cuantitativo (Hernández, Fernández y Baptista, 2010), realizado con una muestra de alumnos de educación básica. El muestreo fue intencional o no probabilístico, en el que participaron los alumnos que se hallaban en las aulas en el momento de la administración de las pruebas.

\subsection{Participantes}

Participó un total 186 estudiantes hispanohablantes de $2^{\circ}$ a $8^{\circ}$ año básico, cuyo nivel de comprensión media fue de 66,35 ( $\mathrm{DE}=18,57)$, de los cuales 95 eran niñas $(\bar{x}=68,59 ; \mathrm{DE}=17,84)$ y 91 niños $(\bar{x}=64,01 ; \mathrm{DE}=19,12)$, que estaban escolarizados en un centro particular subvencionado de la ciudad de Iquique (Chile). El establecimiento, según informe del Mineduc (2016), pertenece a un grupo socioeconómico medio. Su distribución según curso y género se detalla en la Tabla 1.

Previamente se excluyeron de la muestra los estudiantes con discapacidad o dificultades específicas de aprendizaje, según información de sus tutores. Además de cumplirse los principios éticos para la investigación en seres humanos, los niños aceptaron participar 
y entregaron el consentimiento firmado por sus padres al equipo de investigación.

Tabla 1. Tamaño muestral, según nivel de enseñanza y género.

\begin{tabular}{|l|c|c|c|}
\hline Nivel de enseñanza & Niñas & Niños & Total \\
\hline $2^{\circ}$ año básico & 15 & 14 & 29 \\
\hline $3^{\circ}$ año básico & 17 & 10 & 27 \\
\hline $4^{\circ}$ año básico & 11 & 12 & 23 \\
\hline $5^{\circ}$ año básico & 11 & 14 & 25 \\
\hline $6^{\circ}$ año básico & 13 & 15 & 28 \\
\hline $7^{\circ}$ año básico & 15 & 16 & 31 \\
\hline $8^{\circ}$ año básico & 13 & 10 & 23 \\
\hline Total & 95 & 91 & 186 \\
\hline
\end{tabular}

Fuente: Elaboración propia.

\subsection{Instrumentos}

Para conocer el nivel de comprensión lectora del alumnado, se manejaron pruebas no estandarizadas, diseñadas ad hoc para cada nivel escolar, similares a las utilizadas por las evaluaciones Simce que realiza la Agencia de Calidad en Chile (ACE), y que están sustentadas en las lecturas de las Pruebas del Dominio Lector (Marchant, Recart, Cuadrado y Sanhueza, 2004), un instrumento validado en Chile acerca de la velocidad y calidad de la lectura. Se trata de pruebas de selección múltiple (a nivel literal, inferencial y crítico), para asegurar validez, confiabilidad y objetividad, que han sido certificadas por juicio de expertos y triangulación, con un porcentaje de acuerdo del 93\%. Tres investigadores universitarios expertos en didáctica de la lengua y dos profesoras de educación básica, que imparten docencia en la asignatura de Lenguaje y Comunicación, determinaron la validez de su contenido, de acuerdo con criterios de relevancia y representatividad de los ítems (Sireci \& Faukner-Bond, 2014). El grado de concordancia, según el Índice Kappa, fue muy positivo $(\kappa=0,91)$.

Las pruebas constaron de 12 preguntas de selección múltiple a las que se les asignó la puntuación 0 (incorrecta) y 1 (correcta). El formato de las pruebas fue de papel y lápiz. Una muestra de las preguntas para cada nivel de comprensión lectora, según el curso, se recoge en la Tabla 2 . 
Tabla 2. Muestra de preguntas acerca de la comprensión lectora.

\begin{tabular}{|c|c|c|c|}
\hline Nivel & Literal & Inferencial & Crítico \\
\hline $\begin{array}{l}2^{\mathbf{o}} \text { año } \\
\text { básico }\end{array}$ & $\begin{array}{l}\text { ¿Quién entra a la } \\
\text { casa? } \\
\text { a. La paloma. } \\
\text { b. El mago. } \\
\text { c. El hada. }\end{array}$ & $\begin{array}{l}\text { Según el texto, se podría } \\
\text { pensar que la paloma es: } \\
\text { a. observadora. } \\
\text { b. inquieta. } \\
\text { c. miedosa. }\end{array}$ & $\begin{array}{l}\text { El perro esconde un hueso } \\
\text { porque: } \\
\text { a. es juguetón. } \\
\text { b. no le gusta. } \\
\text { c. lo comerá después. }\end{array}$ \\
\hline $\begin{array}{l}3^{\mathbf{0}} \text { año } \\
\text { básico }\end{array}$ & $\begin{array}{l}\text { ¿Por qué lloró } \\
\text { Ricardo? } \\
\text { a. Porque se cayó su } \\
\text { potrillo. } \\
\text { b. Porque se cayó en } \\
\quad \text { un barrial. } \\
\text { c. Porque el potrillo } \\
\text { salió al galope. } \\
\text { d. Porque Luisa se } \\
\text { burló. }\end{array}$ & $\begin{array}{l}\text { ¿Por cuál palabra puede } \\
\text { ser reemplazada la } \\
\text { palabra destacada en la } \\
\text { siguiente oración? } \\
\text { "Un día su padre les } \\
\text { regaló un potrillo alazán" } \\
\text { a. Holgazán. } \\
\text { b. Anaranjado. } \\
\text { c. Joven. } \\
\text { d. Veloz. }\end{array}$ & $\begin{array}{l}\text { La intención del autor de este } \\
\text { texto es: } \\
\text { a. contar lo peligroso que es } \\
\text { tener un potrillo. } \\
\text { b. informar una noticia } \\
\text { sobre unos niños y su } \\
\text { potrillo. } \\
\text { c. contar una historia sobre } \\
\text { unos niños y su potrillo. } \\
\text { d. convencer sobre lo bueno } \\
\text { que es tener un potrillo. }\end{array}$ \\
\hline $\begin{array}{l}4^{\circ} \text { año } \\
\text { básico }\end{array}$ & $\begin{array}{l}\text { ¿Qué es un naranjo? } \\
\text { a. Una fruta. } \\
\text { b. Una flor. } \\
\text { c. Un árbol. } \\
\text { d. Unas raíces. }\end{array}$ & $\begin{array}{l}\text { ¿De quién habla el texto } \\
\text { cuando dice que da } \\
\text { flores blancas y muy } \\
\text { aromáticas? } \\
\text { a. De las flores. } \\
\text { b. De las hojas del } \\
\quad \text { naranjo. } \\
\text { c. De la naranja. } \\
\text { d. Del naranjo. }\end{array}$ & $\begin{array}{l}\text { "La naranja es de color amari- } \\
\text { llo rojizo, de rico sabor y muy } \\
\text { jugosa". Esta frase expresa: } \\
\text { a. hecho y opinión. } \\
\text { b. solo hecho. } \\
\text { c. solo opinión. } \\
\text { d. ni hecho ni opinión. }\end{array}$ \\
\hline $\begin{array}{l}5^{\mathbf{0}} \text { año } \\
\text { básico }\end{array}$ & $\begin{array}{l}\text { Los araucanos tenían } \\
\text { rebaños de: } \\
\text { a. pumas. } \\
\text { b. llamas. } \\
\text { c. guanacos. } \\
\text { d. ovejas. }\end{array}$ & $\begin{array}{l}\text { Según el texto, los arauca- } \\
\text { nos usaban utensilios de } \\
\text { greda y madera. Esto se } \\
\text { refiere a: } \\
\text { a. objetos de cocina y } \\
\text { herramientas. } \\
\text { b. enseres de baño. } \\
\text { c. herramientas para } \\
\text { hacer las rucas. } \\
\text { d. objetos exclusivos } \\
\text { para cultivar. }\end{array}$ & $\begin{array}{l}\text { Según el texto, se podría } \\
\text { pensar que los araucanos: } \\
\text { a. llevaban una vida } \\
\text { nómada. } \\
\text { b. se dedicaban a desarrollar } \\
\text { el arte. } \\
\text { c. se establecieron en } \\
\text { un lugar en forma } \\
\text { permanente. } \\
\text { d. eran grandes guerreros, } \\
\text { enérgicos y tenaces. }\end{array}$ \\
\hline $\begin{array}{l}6^{\circ} \text { año } \\
\text { básico }\end{array}$ & $\begin{array}{l}\text { ¿Qué es la } \\
\text { salamandra? } \\
\text { a. Un anfibio. } \\
\text { b. Una larva. } \\
\text { c. Un tipo de sapo. } \\
\text { d. Un tipo de rana. }\end{array}$ & $\begin{array}{l}\text { ¿A quién se refiere el texto } \\
\text { cuando dice: "en un prin- } \\
\text { cipio, utilizan branquias o } \\
\text { agallas..."? } \\
\text { a. A los anfibios. } \\
\text { b. A las ranas. } \\
\text { c. A las salamandras. } \\
\text { d. A los sapos. }\end{array}$ & $\begin{array}{l}\text { La diferencia entre la sala- } \\
\text { mandra y el sapo es que: } \\
\text { a. la salamandra mantiene } \\
\text { los pulmones y branquias. } \\
\text { b. el sapo mantiene las } \\
\text { branquias y los pulmones. } \\
\text { c. la salamandra mantiene } \\
\text { las branquias y agallas. } \\
\text { d. el sapo mantiene las agal- } \\
\text { las y los pulmones. }\end{array}$ \\
\hline
\end{tabular}




\begin{tabular}{|c|c|c|c|}
\hline $\begin{array}{l}7^{\mathbf{0}} \text { año } \\
\text { básico }\end{array}$ & $\begin{array}{l}\text { Según el texto, ¿cuál } \\
\text { es el recorrido del } \\
\text { agua? } \\
\text { a. De las montañas } \\
\text { a la ribera de los } \\
\text { ríos. } \\
\text { b. De la cordillera a } \\
\text { las siembras. } \\
\text { c. De las piedras al } \\
\text { mar. } \\
\text { d. De las montañas } \\
\text { al mar. }\end{array}$ & $\begin{array}{l}\text { ¿Qué significa que los ríos } \\
\text { sean caudalosos? } \\
\text { a. Que arrastran tierra y } \\
\text { piedras. } \\
\text { b. Que nacen de } \\
\text { los deshielos } \\
\text { cordilleranos. } \\
\text { c. Que van de la cordil- } \\
\text { lera al mar. } \\
\text { d. Que tienen mucha } \\
\text { agua. }\end{array}$ & $\begin{array}{l}\text { ¿Cuál de los siguientes signi- } \\
\text { ficados de la palabra erosión } \\
\text { tiene relación con el texto } \\
\text { leído? } \\
\text { a. Desgaste producido en la } \\
\text { superficie de un cuerpo } \\
\text { por fricción continua de } \\
\text { otro. } \\
\text { b. Lesión superficial de la } \\
\text { epidermis, producida por } \\
\text { un agente externo. } \\
\text { c. Desgaste de prestigio o in- } \\
\text { fluencia que puede sufrir } \\
\text { una persona o institución. } \\
\text { d. Recorrido del caudal de } \\
\text { los ríos. }\end{array}$ \\
\hline $\begin{array}{l}8^{\mathbf{o}} \text { año } \\
\text { básico }\end{array}$ & $\begin{array}{l}\text { ¿Qué ocurre primero } \\
\text { en la historia? } \\
\text { a. Va camino a su } \\
\text { casa. } \\
\text { b. Blanca está } \\
\text { indecisa. } \\
\text { c. Los padres la } \\
\text { animan a que } \\
\text { elija su mascota. } \\
\text { d. Se le ocurre una } \\
\text { idea. }\end{array}$ & $\begin{array}{l}\text { ¿Qué problema tenía } \\
\text { Blanca? } \\
\text { a. No tenía mascota. } \\
\text { b. No sabía a cuál de sus } \\
\quad \text { mascotas elegir. } \\
\text { c. Temía decepcionar a } \\
\quad \text { sus amigas. } \\
\text { d. Temía no encontrar } \\
\quad \text { una araña "pollito". }\end{array}$ & $\begin{array}{l}\text { La intención del autor de este } \\
\text { texto es: } \\
\text { a. Convencer sobre lo } \\
\text { importante que es tener } \\
\text { amigas. } \\
\text { b. Contar sobre el } \\
\text { cumplimiento de las } \\
\text { promesas. } \\
\text { c. Contar una historia } \\
\text { sobre la influencia de las } \\
\text { amigas. } \\
\text { d. Convencer sobre lo bueno } \\
\text { que es tener mascota. }\end{array}$ \\
\hline
\end{tabular}

Fuente: Elaboración propia.

El empleo de preguntas de selección múltiple de alternativas es uno de los procedimientos más empleados para evaluar la comprensión lectora. Consiste en proporcionar "a los alumnos un texto relativamente corto seguido de varias preguntas que, a su vez, tienen respuestas posibles de entre las cuales el alumno debe elegir la que considere correcta" (PérezZorrilla, 2005, p. 128).

\subsection{Procedimiento}

Las pruebas se administraron durante una jornada escolar y de forma colectiva en cada nivel de enseñanza en la sala de clase correspondiente, durante 2017. Además de la investigadora principal, colaboraron estudiantes del último año de Psicopedagogía, previamente instruidas para asegurar su correcta aplicación. Se informó al alumnado respecto del anonimato de las respuestas y de sus condiciones. Por medio de un ejemplo, se les explicó preliminarmente la forma de contestar en una hoja de 
respuesta, para asegurar su comprensión. La duración de la aplicación de la prueba fue de 45 a 70 minutos.

A partir de las respuestas, y de acuerdo con los informes de resultados Simce (2016) que realiza la ACE, el alumnado se distribuyó en tres grupos, atendiendo a su nivel de aprendizaje lector, para clasificar el logro alcanzado por los estudiantes, como se hace en las evaluaciones nacionales según: nivel insuficiente, en el que se incluyen los alumnos con menos del $60 \%$ de respuestas correctas; nivel elemental, en el que se registran los que logran entre 60 y $79 \%$ de respuestas correctas; y nivel adecuado, en el que aparecen quienes logran $80 \%$ o más de respuestas correctas.

Por otra parte, se obtuvo el promedio de los porcentajes de respuestas correctas en función del nivel de comprensión lectora: literal, que incluye preguntas que los alumnos son capaces de comprender de forma muy básica; inferencial, en el que se incluyen preguntas que permiten realizar conjeturas e hipótesis sobre el texto; crítico, referido a las habilidades para reflexionar sobre lo que se lee, establecer relaciones y emitir opiniones argumentadas acerca de la calidad del texto.

\subsection{Análisis estadístico}

Con la ayuda del IBM SPSS (v. 22.0), se realizaron análisis descriptivos e inferenciales. Se obtuvieron frecuencias y porcentajes según las variables estudiadas, procurando identificar las posibles diferencias significativas.

Para analizar la significatividad de las diferencias entre cursos, se aplicó un ANOVA y se realizaron comparaciones post hoc mediante el procedimiento Bonferroni, para comprobar entre qué cursos se verificaban esas diferencias. Las discrepancias por género para el grupo total se hallaron con el estadístico paramétrico $t$ de Student y con la prueba no paramétrica U de Mann-Whitney, en el caso de cada curso o nivel de enseñanza, dado que el test de Levene desaconsejó el uso de una prueba paramétrica en los niveles escolares evaluados. En todas las pruebas se estableció como criterio de significatividad un valor menor a 0,05 .

\section{Resultados}

Como se observa en la Tabla 3, el desempeño del alumnado en tareas de comprensión lectora se empobrece a partir de $4^{\circ}$ año básico, existiendo 
diferencias significativas entre cursos, según los puntajes de logro, como se ha puesto de manifiesto con el ANOVA ( $F=11,478 ; p<0,001 ; \mathrm{y} \mathrm{GL} 6 ; 179)$. El tamaño del efecto es medio $(d=0,5)$. La comparación post hoc entre niveles de enseñanza mediante el procedimiento Bonferroni arrojó diferencias significativas entre $2^{\circ}$ y $4^{\circ}$ año $(p<0,001)$, entre $2^{\circ}$ y $5^{\circ}$ año $(p$ $<0,001)$ y entre $2^{\circ}$ y $7^{\circ}$ año $(p<0,001)$, siempre a favor del alumnado de $2^{\circ}$ año lo que significa que la comprensión lectora de los estudiantes de $4^{\circ}, 5^{\circ}$ y $7^{\circ}$ año es inferior a la de los niños de $2^{\circ}$ año básico. También se apreciaron diferencias entre $3^{\circ}$ y $7^{\circ}$ año $(p<0,001)$, entre $6^{\circ}$ y $7^{\circ}$ año $(p<$ o,001), cuyos niveles de aprendizaje han sido más altos también en los cursos inferiores. Sin embargo, las diferencias significativas halladas entre $7^{\circ}$ y $8^{\circ}$ año $(p<0,001)$ revelan que son los estudiantes del curso superior quienes obtienen mejor puntaje. Llama la atención que sea el alumnado de $7^{\circ}$ año el que obtenga el puntaje más bajo y el de $2^{\circ}$ año el más alto, con una diferencia entre ambos de 30,9 puntos.

Tabla 3. Resultados de la evaluación de la comprensión lectora.

\begin{tabular}{|l|c|c|c|c|c|c|}
\hline \multirow{2}{*}{$\begin{array}{l}\text { Nivel de } \\
\text { enseñanza }\end{array}$} & \multicolumn{2}{|c|}{ Niñas } & \multicolumn{2}{c|}{ Niños } & \multicolumn{2}{c|}{ Promedio curso } \\
\hline & $\%$ RC & DE & \% RC & DE & \% RC & DE \\
\hline $\mathbf{2}^{\mathbf{o}}$ año básico & 83,90 & 13,90 & 79,81 & 19,50 & 81,90 & 16,68 \\
\hline $\mathbf{3}^{\mathbf{0}}$ año básico & 71,11 & 19,82 & 70,89 & 20,12 & 70,99 & 19,52 \\
\hline $\mathbf{4}^{\mathbf{0}}$ año básico & 62,90 & 12,04 & 54,23 & 22,30 & 58,33 & 18,29 \\
\hline $\mathbf{5}^{\mathbf{0}}$ año básico & 67,42 & 16,90 & 57,21 & 14,21 & 61,67 & 15,95 \\
\hline $\mathbf{6}^{\mathbf{0}}$ año básico & 68,67 & 13,22 & 69,52 & 16,62 & 69,05 & 14,85 \\
\hline $\mathbf{7}^{\mathbf{0}}$ año básico & 53,01 & 17,51 & 49,50 & 12,02 & 51,07 & 14,39 \\
\hline $\mathbf{8}^{\mathbf{0}}$ año básico & 72,41 & 11,03 & 70,80 & 12,01 & 71,73 & 11,16 \\
\hline Total & $\mathbf{6 8 , 5 9}$ & $\mathbf{1 7 , 8 4}$ & $\mathbf{6 4 , 0 1}$ & $\mathbf{1 9 , 1 2}$ & $\mathbf{6 6 , 3 5}$ & $\mathbf{1 8 , 5 7}$ \\
\hline
\end{tabular}

Nota: \% RC: Porcentaje medio de respuestas correctas; DE: Desviación estándar. Fuente: Elaboración propia.

Respecto del género, no se han hallado diferencias significativas en el nivel de comprensión lectora entre el grupo de niñas y el de niños. Comparando el grupo total, el estadístico $t$ de Student reveló la inexistencia de diferencias $(t=0,04, p=0,84)$. Confrontando ambos grupos (niñas y niños), según el nivel de enseñanza, tampoco se apreciaron diferencias significativas entre los cursos, como demostró la prueba U de Mann-Whitney (en $2^{\circ}$ año, $p=0,56$; en $3^{\circ}$ año, $p=0,94$; en $4^{\circ}$ año, $p=0,44$; en $5^{\circ}$ año, $p=0,85$; en $6^{\circ}$ año, $p=0,33$; en $7^{\circ}$ año, 
$p=0,95$; en $8^{\circ}$ año, $p=0,60$ ). Se ha detectado, no obstante, que en el grupo de los niños la desviación estándar es ligeramente mayor que en el de las niñas.

Teniendo en cuenta el nivel de enseñanza o el curso y los porcentajes de respuestas correctas obtenidos por el alumnado en las pruebas de comprensión lectora, se muestra la tendencia negativa que siguen estos estudiantes (Figura 1), en cuanto al nivel de logro que consiguen. Como puede apreciarse, sorprende que el nivel de comprensión lectora no avance progresivamente cuando el alumnado se halla escolarizado en el siguiente nivel de enseñanza, advirtiéndose resultados muy similares entre el grupo de niños y el de niñas.

Figura 1. Tendencia en el promedio de \%RC de comprensión lectora.

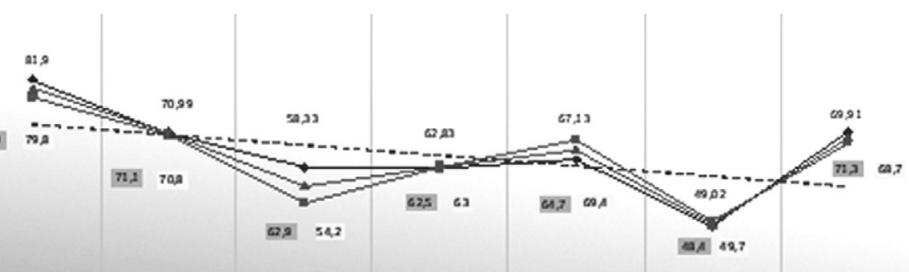

28

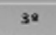

se

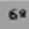

78

Fuente: Elaboración propia.

Asimismo, según el nivel de enseñanza, en la Figura 2 se incluye el porcentaje de respuestas correctas obtenido en cada uno de los niveles de comprensión lectora. En general, el comportamiento es similar entre los cursos y los mejores desempeños del alumnado en lectura se aprecian, como era presumible, en el nivel literal, seguido del inferencial $\mathrm{y}$, posteriormente del crítico. No obstante, la tendencia se invierte en $4^{\circ}$ año básico, donde el porcentaje de respuestas correctas es levemente inferior a nivel inferencial que a nivel crítico, y en $8^{\circ}$ año, donde la comprensión inferencial es superior a la literal. Nuevamente es entre $2^{\circ}$ y $7^{\circ}$ año básico donde se observan las mayores diferencias en el nivel crítico, con 60 puntos porcentuales. 
Figura 2. Porcentaje de respuestas correctas según niveles de enseñanza y de comprensión lectora.

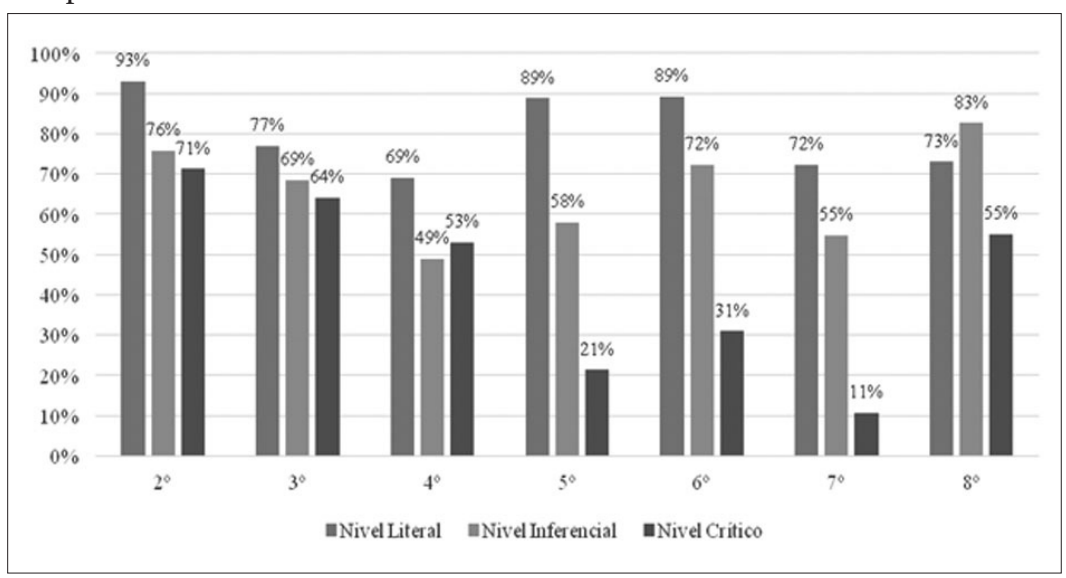

Fuente: Elaboración propia.

Finalmente, a partir del nivel de enseñanza o curso de escolaridad, en la Figura 3 se muestra el porcentaje de estudiantes según el nivel de aprendizaje conseguido en comprensión lectora. Se podrá apreciar que el nivel de logro insuficiente es superior al de $2^{\circ}$ año en todos los cursos y de manera muy significativa en $4^{\circ}, 5^{\circ}$ y $7^{\circ}$ año básico. Podría decirse que menos de la mitad del alumnado de los cursos de $4^{\circ}$ y $5^{\circ}$ año son capaces de comprender un texto, así como también que solo un 8,7\% del alumnado de $4^{\circ}$ año y un $20 \%$ de los estudiantes de $5^{\circ}$ año son lectores competentes. Más llamativos aún son los datos cuando se comparan $2^{\circ}$ y $7^{\circ}$ año: apenas alcanza un nivel de aprendizaje adecuado el $3,2 \%$ en $7^{\circ}$ año, frente al $69 \%$ en $2^{\circ}$ año y un nivel elemental del $16,1 \%$ en $7^{\circ}$ año, frente al $17,2 \%$ en $2^{\circ}$ año. También sorprende que los estudiantes de $8^{\circ}$ año, al concluir la escolaridad básica, alcancen solo un nivel de aprendizaje adecuado del $26,1 \%$ frente al conseguido por el alumnado de $3^{\circ}$ año (37\%) o de $2^{\circ}$ año (69\%). 
Figura 3. Porcentaje de estudiantes según niveles de aprendizaje lector.

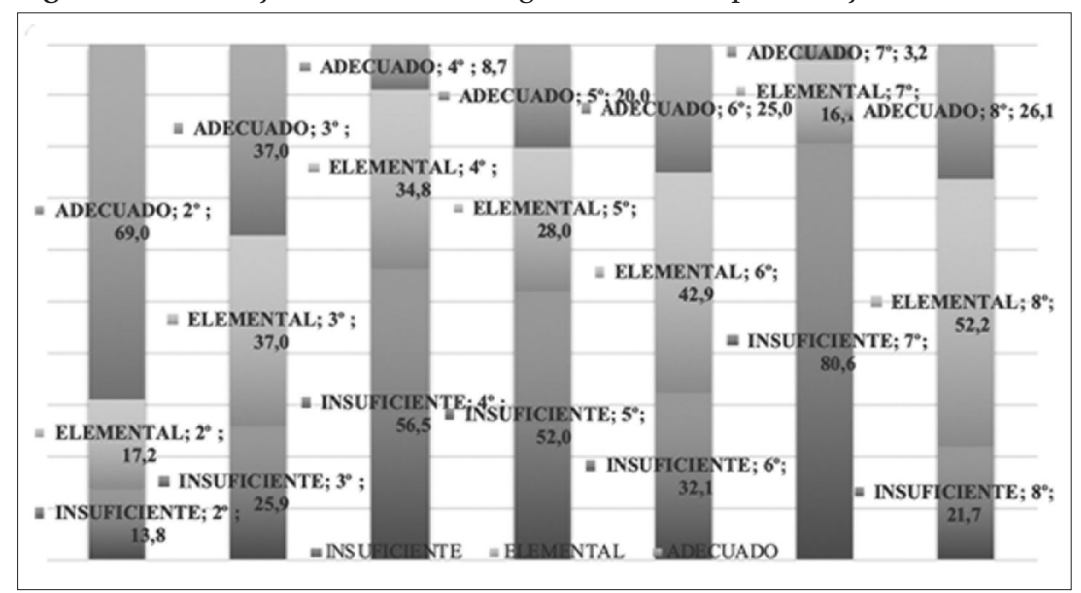

Fuente: Elaboración propia.

Ahora bien, si se compara el informe Simce de 2015 con los hallazgos de nuestro estudio, en el nivel de $2^{\circ}$ año básico se aprecian mayores diferencias entre ambos estudios, en cuanto a los niveles de aprendizaje: en el nivel insuficiente (18,3\% frente al 13,8\%), en el nivel elemental ( $45 \%$ frente al $17,2 \%$ ) y en el nivel adecuado (36,7\% frente al $69 \%$ ). La comparación no es posible con el informe Simce de 2016, ya que en esa oportunidad no se evaluó $2^{\circ}$ año básico.

En $4^{\circ}$ año básico, comparando el informe Simce de 2015 y los resultados de nuestra investigación, los datos son similares en los niveles insuficiente (49,1\% frente al 56,5\%) y elemental (29,1\% frente al $34,8 \%$ ), pero no en el nivel adecuado (21,8\% frente al 8,7\%). Por su parte, en el informe Simce de 2016 —en el que se volvió a evaluar $4^{\circ}$ básico—, los datos de ambos estudios son más discrepantes en el nivel insuficiente $(32,3 \%$ frente al $56,5 \%)$ y elemental $(24,6 \%$ frente al $34,8 \%)$, y de forma muy especial en el nivel adecuado $(43,1 \%$ frente al $8,7 \%)$.

Si se toma como referente el $8^{\circ}$ año básico, al comparar el informe Simce de 2015 con los datos de nuestra investigación se observa que en el nivel elemental el porcentaje es casi idéntico ( $50 \%$ frente al 52,2\%), aunque no así en los niveles insuficiente (36,5\% frente al 21,7\%) y adecuado (13,5\% frente al $26,1 \%$ ). La comparación no es posible con el informe Simce de 2016, ya que no se evaluó $8^{\circ}$ año básico en esa oportunidad. 


\section{Discusión y conclusiones}

El objetivo de este estudio fue indagar en el nivel de comprensión lectora de estudiantes chilenos de $2^{\circ}$ a $8^{\circ}$ año de enseñanza básica, en las variables definidas en la literatura científica como explicativas de la comprensión lectora, así como en el nivel de logro establecido por el Simce, teniendo en cuenta el género de los estudiantes. Los resultados han puesto de manifiesto que no existen diferencias significativas entre niñas y niños, aunque pudo comprobarse que existen resultados disímiles según el curso de escolaridad e importantes diferencias en los niveles de comprensión lectora, siendo los estudiantes de $4^{\circ}$ y de $7^{\circ}$ año básico quienes obtuvieron los rendimientos más bajos. Estos hallazgos son preocupantes por cuanto la competencia lectora es determinante para el éxito escolar (Bravo, Villalón y Orellana, 2002; Fajardo, Hernández y González, 2012).

Por el contrario, los alumnos de $2^{\circ}, 3^{\circ}$ y $8^{\circ}$ año básico, respectivamente, son quienes lograron los mayores niveles de competencia lectora, especialmente el alumnado de $2^{\circ}$ año básico. Estos datos son consistentes con los resultados del informe Simce de 2015, en el que los alumnos de este nivel obtuvieron mejores niveles de aprendizaje lector adecuado, aunque las discrepancias entre ambos estudios son manifiestas en cuanto a los porcentajes de logro obtenidos por los escolares en los niveles elemental e insuficiente.

Sin embargo, la distribución por niveles de aprendizaje en los estudiantes de $4^{\circ}$ año básico (nivel evaluado en 2015 junto con $2^{\circ}$ y $8^{\circ}$ año básico) es similar comparando el informe Simce de 2015 y los resultados de nuestra investigación. No obstante, en $8^{\circ}$ año básico se observaron diferencias en los niveles insuficiente y adecuado, pero no en el nivel elemental, donde el porcentaje es casi idéntico. Pero la distribución análoga que se observa en algunos niveles de aprendizaje (en $2^{\circ}$ y $4^{\circ}$ año en el nivel insuficiente y en $4^{\circ}$ y $8^{\circ}$ año en el nivel elemental), en ambos estudios, podría hacer pensar que el profesorado reproduce un esquema-modelo de estrategias de enseñanza en tareas lectoras que se mantiene a lo largo de la etapa escolar.

Por otra parte, se ha podido apreciar que el alumnado de $5^{\circ}$ y $7^{\circ}$ año básico obtiene un rendimiento lector más bajo en el nivel inferencial. Este hallazgo resulta trascendental, si se tiene en cuenta que las inferencias desempeñan un papel relevante para comprender el mundo circundante (Dubois, 1984). Al respecto, algunos autores sitúan las inferencias como 
un eje en el proceso de comprensión lectora y aconsejan la enseñanza de estrategias inferenciales para ahondar en la comprensión de los textos y superar la comprensión literal de los escritos (Cain \& Oakhill, 1999; Jouini y Saud, 2005). A través de las inferencias, el lector podrá relacionar experiencias y vivencias propias con las halladas en el texto, lo que le permitirá hacer conjeturas y establecer hipótesis acerca de los contenidos de la lectura, evitando así obtener una información desconectada y poco comprensible (Graesser, Singer \& Trabasso, 1994; Pérez-Zorrilla, 2005).

Asimismo, los resultados del estudio revelaron cómo el nivel de competencia lectora decrece a medida que los estudiantes avanzan de curso escolar, lo cual contrasta con los datos obtenidos por Andrés, Urquijo, Navarro y Canet (2014), donde se aprecia que existe una mayor capacidad de comprensión lectora conforme se avanza en la escolaridad. Esto pudiera deberse, tal vez, a una cierta despreocupación del profesorado por la lectura en los niveles superiores, por entender que el aprendizaje lecto-escritor demanda una mayor dedicación en los niveles iniciales ( $2^{\circ}$ año básico) que en los superiores $\left(7^{\circ}\right.$ y $8^{\circ}$ año básico), y también a no considerar suficientemente el incremento de la complejidad léxica que poseen los textos en los cursos superiores. Algunos estudios han puesto de manifiesto cómo el profesorado de los niveles más básicos parece mostrar una mayor preocupación por el aprendizaje de la lectura (Galdames, Medina, San Martín, Gaete y Valdivia, 2011).

Se ha señalado que a partir de $4^{\circ}$ año básico se incrementan las dificultades del alumnado para responder a cuestiones que requieren establecer relaciones complejas entre unidades de información que han de ser activadas desde el conocimiento previo (De Mier, Borzone y Cupani, 2012; De Mier, Borzone, Sánchez, y Benítez, 2013). Al respecto, se han subrayado los escasos desafíos orales que los profesores plantean en las actividades de aprendizaje, dado que tienden a reiterar "un mismo tipo de desafío cognitivo en todos los momentos de la clase y frente a todo tipo de actividades" (Medina, Valdivia, Gaete, y Galdames, 2015, p. 195).

Finalmente, los resultados de nuestro estudio evidencian un manejo insuficiente de las estrategias de autorregulación del proceso lector, algo muy común en los lectores menos competentes, quienes podrían utilizar estas estrategias de forma adecuada si recibiesen instrucción precisa (Gutiérrez y Salmerón, 2012; Irrazabal, 2007). De hecho, la interacción docente-estudiantes resulta trascendental en la enseñanza de la lectura 
y la manera en que el profesorado proporciona las oportunidades para favorecer las habilidades con el lenguaje oral y escrito, para que los escolares puedan enfrentarse a los retos cognitivos que el aprendizaje de la lectura requiere (Medina et al., 2015).

Por último, si comprender un texto es algo más que una tarea de decodificación grafema-fonema y de reconocimiento de palabras, pues exige poner en funcionamiento estrategias de alto nivel cognitivo durante la interacción lector-texto-contexto, para inferir significados, reflexionar sobre las ideas vertidas y emitir opiniones acerca de los aspectos más relevantes del texto, la enseñanza del proceso lector tiene implicaciones a nivel pedagógico, que afectan la acción de los docentes.

En este sentido, teniendo en cuenta los resultados de este estudio y en sintonía con la literatura especializada (Ahmadi, 2012; Gutiérrez, 2016; Gutiérrez, y Salmerón, 2012; Rosas, Escobar, Ramírez, Meneses, y Guajardo, 2017, entre otros), se sugiere la implementación de diferentes tareas para mejorar la comprensión lectora de los estudiantes, entre las que se pueden nombrar las siguientes: elaboración de hipótesis, inferencias y conjeturas, tareas de entrenamiento en habilidades fonológicas, actividades que conlleven la evocación de conocimientos previos, la tipificación de los aspectos relevantes de un texto, interpretación personal de diferentes tipos de textos, el manejo de organizadores gráficos, la reflexión en torno a las ideas principales de un texto, la lectura dialógica, la puesta en común de las estrategias lectoras, realización de tareas de lectura silenciosa y en voz alta, relectura selectiva de textos, subrayado de ideas focales y elaboración de resúmenes.

Este estudio, sin embargo, tiene sus limitaciones. Por ejemplo, los resultados se circunscriben a un único escenario (centro urbano chileno); la valoración de la comprensión lectora fue realizada únicamente con pruebas no estandarizadas; y la muestra de estudiantes pertenece a un solo nivel socioeconómico. Por ello, se hacen necesarios nuevos estudios que exploren otros contextos (rurales), que manejen pruebas estandarizadas y diferentes tipologías textuales, así como también podrían incorporarse estudiantes de grupos socioeconómicos distintos a la muestra. Conocer cuáles son las dificultades específicas para la lectura de los estudiantes de un establecimiento educativo concreto es un excelente punto de partida para diseñar estrategias de intervención in situ que puedan paliar esos problemas. 


\section{Referencias bibliográficas}

Ahmadi, M. (2012). Impacts of learning reading strategy on student's reading comprehension proficiency. The International Journal of Language and Applied Linguistics World, 1(1), 72-87.

Alonso, J., Carriedo, N., González, E., Gutiérrez, F., y Mateos, M. M. (1992). Leer, comprender y pensar: nuevas estrategias y técnicas de evaluación. Madrid: Centro de Publicaciones del Ministerio de Educación y Ciencia, CIDE.

Alliende, F. y Condemarín, M. (2002). La lectura: teoría, evaluación y desarrollo. Santiago de Chile: Andrés Bello.

Andrés, M. L., Urquijo, S., Navarro, J. I., y Canet, L. (2014). Relación de las habilidades metalingüísticas con la adquisición y consolidación de la lectura. Revista de Psicología y Educación, 9(1), 71-84. Recuperado de https://www.aacademica.org/ sebastian.urquijo/119.pdf

Best, R. M., Floyd, R. G., \& McNamara, D. S. (2008). Differential competencies contributing to children's comprehension of narrative and expository texts. Reading Psychology, 29(2), 137-164. https://doi. org/10.1080/02702710801963951

Bigozzi, L., Tarchi, C., Vagnoli, L., Valente, E., \& Pinto, G. (2017). Reading fluency as a predictor of school outcomes across grades 4-9. Frontiers in Psychology, 8, 1-9. https://doi.org/10.3389/ fpsyg.2017.00200

Bohórquez, L. F., Cabal, M. A., y Quijano, M. C. (2014). La comprensión verbal y la lectura en niños con y sin retraso lector. Revista Pensamiento Psicológico, 12(1), 169-182. https://doi. org/10.11144/javerianacali.ppsi12-1.cvln

Bravo, L., Villalón, M., y Orellana, E. (2002). La conciencia fonológica y la lectura inicial en niños que ingresan a primer año básico. Psykhé, 11(1), 175-182. Recuperado de http://www.psykhe.cl/ index.php/psykhe/article/view/452

Cain, K. \& Oakhill, J. V. (1999). Inference making ability and its relation to comprehension failure in young children. Reading and Writing, 11(5-6), 489-503. https://doi.org/10.1023/A:1008084120205

Couceiro, A. P. \& Botelho, A. R. (2017). Consciência fonológica e desempenho geral na leitura. Que relação? Estudo com alunos dos $2^{\circ}$ 
e $3^{\circ}$ anos de escolaridade. Revista Praxis Educativa, 12(1), 48-63. https://doi.org/10.5212/praxeduc.v.12i1.0003

De la Calle, A. M., Villagrán, M. A., y Guzmán, J. I. N. (2016). Desarrollo evolutivo de la conciencia fonológica: ¿Cómo se relaciona con la competencia lectora posterior? Revista de Investigación en Logopedia, 6(1), 22-41. Recuperado de https://revistas.ucm.es/ index.php/RLOG/article/download/58553/52683

De Mier, M. V., Borzone, A. M., y Cupani, M. (2012). La fluidez lectora en los primeros grados: relación entre habilidades de decodificación, características textuales y comprensión. Un estudio piloto con niños hablantes de español. Revista Neuropsicología Latinoamericana, 4(1), 18-33. Recuperado de http://www.redalyc.org/articulo.oa?id=439542720003

De Mier, M. V., Borzone, A. M., Sánchez, V. S., y Benítez, M. E. (2013). Habilidades de comprensión y factores textuales en los primeros grados. Revista Peruana de Psicología y Trabajo Social, 2(1), 89-106. http://dx.doi.org/www.uigv.edu.pe/ psicologia-y.../revista-peruana-de-psicologia-y-trabajo

Dubois, M. E. (1984). Algunos interrogantes sobre comprensión de la lectura. Revista Lectura y Vida, 5(4), 14-19. Recuperado de http://www.lecturayvida.fahce.unlp.edu.ar/numeros/ a5n4/05_04_Dubois.pdf

Dreyer, C. \& Nel, C. (2013). Teaching reading strategies and reading comprehension within a technology-enhanced learning environment. System, 31(3), 349-365. https://doi.org/10.1016/ s0346-251x(03)00047-2

Fajardo, A., Hernández, J., y González, Á. (2012). Acceso léxico y comprensión lectora: un estudio con jóvenes universitarios. Revista Electrónica de Investigación Educativa, 14(2), 25-33. Recuperado de http://redie.uabc.mx/vol14no2/contenidofajardoetal.html

Galdames, V., Medina, L., San Martín, E., Gaete, R., y Valdivia, A. (2011) ¿Qué actividades realizan los docentes de NB1 para enseñar a leer en situación de evaluación docente? Enfoques tras las prácticas docentes. En J. Manzi, R. González, e Y. Zun (Eds.), La evaluación docente en Chile (pp. 200-203). Santiago de Chile: Ministerio de Educación de Chile/Pontificia Universidad Católica de Chile. 
González, S. M. y López, A. J. (2015). Escolarización temprana: su influencia sobre la comprensión lectora en primaria. Revista Cultura y Educación, 27(2), 237-270. https://doi.org/10.1080/ $\underline{11356405.2015 .1034533}$

Goswami, U. (2011). A temporal sampling framework for developmental dyslexia. Trends in Cognitive Sciences, 15, 3-10. https://doi. org/10.1016/j.tics.2010.10.001

Graesser, A. C., Singer, M., \& Trabasso, T. (1994). Constructing inferences during narrative text comprehension. Psychological Review, 101(3), 371-395. https://doi.org/10.1037//0033-295x.101.3.371

Gutiérrez, R. (2016). La lectura dialógica como medio para la mejora de la comprensión lectora. Revista Investigaciones Sobre Lectura, 5, 52-58. Recuperado de https://riuma.uma.es/xmlui/bitstream/ handle/10630/10948/109-343-1-PB.pdf?sequence=1

Gutiérrez, C. y Salmerón, H. (2012). Estrategias de comprensión lectora: enseñanza y evaluación en educación primaria. Revista Profesorado, 16(1), 183-202. Recuperado de http://www. redalyc.org/articulo.oa?id=56724377011

Guzmán, B., Véliz, M., y Reyes, F. (2017). Memoria operativa, comprensión lectora y rendimiento escolar. Revista Literatura y Lingüistica, 35, 379-404. https://doi.org/10.29344/0717621x.35.1432

Hernández, R., Fernández, C., y Baptista, M. P. (2010). Metodología de la investigación ( $5^{\mathrm{a}}$ ed.). México: McGraw-Hill.

Irrazabal, N. (2007). Metacomprensión y comprensión lectora. Revista Subjetividad y Procesos Cognitivos, 10, 43-60. Recuperado de http://www.redalyc.org/articulo.oa?id=339630249003

Jiménez, E. (2014). Comprensión lectora vs competencia lectora: qué son y qué relación existe entre ellas. Revista Investigaciones Sobre Lectura, 1, 65-74. Recuperado de https://www. comprensionlectora.es/revistaisl/index.php/revistaISL/article/ view $/ 17$

Jouini, K. y Saud, K. (2005). Estrategias inferenciales en la comprensión lectora. Glosas Didácticas. Revista Electrónica Internacional, 13, 95-114. Recuperado de https://www.um.es/glosasdidacticas/ GD13/GD13 10.pdf

Jurado, F., Sánchez, L., Cerchiaro, E., y Paba, C. (2013). Práctica pedagógica y lengua escrita: una búsqueda de sentido. Revista 
Folios, 37, 17-25. https://doi.org/10.17227/01234870.37foli os17.25

Marchant, T., Recart, I., Cuadrado, B., y Sanhueza, J. (2004). Pruebas de dominio lector Fundar para alumnos de enseñanza básica. Santiago de Chile: Ediciones de la Universidad Católica de Chile.

Mariángel, S. V. y Jiménez, J. E. (2016). Desarrollo de la conciencia sintáctica y fonológica en niños chilenos: un estudio transversal. Revista Latinoamericana de Psicología, 48(1), 1-7. https://doi.org/10.1016/j.rlp.2015.09.010

Medina, L., Valdivia, A., Gaete, R., y Galdames, V. (2015). ¿Cómo enseñan a leer los profesores de $1^{\circ}$ y $2^{\circ}$ básico en un contexto de evaluación de desempeño docente en Chile? Revista Estudios Pedagógicos, 41(1), 183-198. https://doi.org/10.4067/ s0718-07052015000100011

Ministerio de Educación de Chile, Mineduc (2012). Lenguaje y comunicación. Programa de estudio para segundo año básico. Unidad de Currículum. Santiago de Chile: Autor.

Ministerio de Educación de Chile, Mineduc (2014). Estándares de aprendizaje. Lectura $2^{\circ}$ básico. Santiago de Chile: Autor.

Ministerio de Educación de Chile, Mineduc (2016). Evaluación de resultados de aprendizaje Simce en comprensión lectora. Recuperado de http://www.agenciaeducacion.cl/

Neira, A. C., Reyes, F., y Riffo, B. E. (2015). Experiencia académica y estrategias de comprensión en estudiantes universitarios de primer año. Revista Literatura y Lingüística, 31, 221-244. https:// doi.org/10.4067/s0716-58112015000100012

Organisation for Economic Co-operation and Development, OECD (2015). PISA 2015: Draft Reading Literacy Framework. Paris: Autor.

Outón, P. y Suárez, A. (2011). Las dificultades de exactitud y velocidad lectoras en escolares de segundo de educación primaria. Revista de Investigación en Educación, 9(2), 153-161. Recuperado de http://hdl.handle.net/10347/16788

Pérez-Zorrilla, M. J. (1998). Evaluación de la comprensión lectora en alumnos de doce años. (Tesis doctoral inédita). Universidad Complutense de Madrid, Madrid. 
Pérez-Zorrilla, M. J. (2005). Evaluación de la comprensión lectora: dificultades y limitaciones. Revista de Educación, número extraordinario, 121-138.

Perfetti, C. \& Stafura, J. (2014). Word knowledge in a theory of reading comprehension. Scientific Studies of Reading, 18(1), 22-37. https://doi.org/10.1080/10888438.2013.827687

Ramírez, P., Rossel, K., y Nazar, G. (2016). Comprensión lectora y metacognición: análisis de las actividades de lectura en dos textos de estudio de la asignatura de Lenguaje y Comunicación de séptimo año básico. Revista Estudios Pedagógicos (Valdivia), 42(2), 213-231. https://doi.org/10.4067/ s0718-07052016000200013

Riffo, B., Reyes, F., Novoa, A., Véliz, M., y Castro, G. (2014). Competencia léxica, comprensión lectora y rendimiento académico en estudiantes de enseñanza media. Revista Literatura y Lingüística, 30, 136-165. https://doi.org/10.4067/ s0716-58112014000200009

Rodríguez, B. A., Calderón, M. E., Leal, M. E., y Arias, N. (2016). Uso de estrategias metacomprensivas para el fortalecimiento de la comprensión lectora en estudiantes de segundo ciclo de un colegio oficial en Bogotá, Colombia. Revista Folios, 1(44), 91108. https://doi.org/10.17227/01234870.44folios93.108

Rosas, R., Escobar, J. P., Ramírez, M. P., Meneses, A., y Guajardo, A. (2017). Impacto de una intervención basada en ordenador en niños chilenos con riesgo de manifestar dificultades lectoras. Revista Infancia y Aprendizaje, 40(1), 158-188. https://doi.org /10.1080/02103702.2016.1263451

Sistema Nacional de Evaluación de Resultados de Aprendizaje, Simce (2015). Resultados nacionales. Santiago de Chile: Ministerio de Educación.

Sistema Nacional de Evaluación de Resultados de Aprendizaje, Simce (2016). Resultados nacionales. Santiago de Chile: Ministerio de Educación.

Sireci, S. \& Faulkner-Bond, M. (2014). Validity evidence based on test content. Psicothema, 26(1), 100-107. https://doi. org/10.4135/9780857025753.n227 
Smith, C. B. (1989). La enseñanza de la lecto-escritura: un enfoque interactivo. Madrid: Aprendizaje Visor.

Van den Broeck, W., Geudens, A., \& Van den Bos, K. P. (2010). The nonword-reading deficit of disabled readers: A developmental interpretation. Developmental Psychology, 46(3), 717-734. https://doi.org/10.1037/a0019038

Vygotsky, L. S. (1979). El desarrollo de los procesos básicos superiores. Barcelona: Grijalbo.

Wise, N., D'Angelo, N., \& Chen, X. (2016). A school-based phonological awareness intervention for struggling readers in early French immersion. Reading and Writing, 29(2), 183-205. https://doi. org/10.1007/s11145-015-9585-9 